\title{
Editorial Collaborators
}

The editors wish to thank the following referees and guest editors of theme issues who have generously given their time and skills to the Journal of Official Statistics during the period 1 October 2019 to 30 September 2020. An asterisk indicates that the referee served more than once during the period.

Abe, Naohito, Hitotsubashi Daigaku Institute of Economic Research, Tokyo, Japan Abel, Guy, Asian Demographic Research Institute, Shanghai, China

Abraham, Katharine, Joint Program in Survey Methodology, College Park, Maryland, U.S.A.

Alaimo Di Loro, Pierfrancesco, La Sapienza - University of Rome, Rome, Italy

Ali, Sajid, Bocconi University, Milan, Italy

Andersson, Per Gösta, Stockholm University, Stockholm, Sweden*

Antoun, Christopher, University of Michigan, Ann Arbor, Michigan, U.S.A.

Argerich, Luis, University of Buenos Aires, Buenos Aires, Argentina

Arora, Sanjay, Ernst and Young LLP, Washington, D.C., U.S.A.

Ashmead, Robert, Ohio Colleges of Medicine Government Resource Center, Columbus,

Ohio, U.S.A.

Axelson, Martin, Statistics Sweden, Örebro, Sweden

Bacchini, Fabio, Italian National Institute of Statistics, Rome, Italy*

Baffour, Bernard, University of Queensland, Brisbane, Australia*

Bakker, Bart, Statistics Netherlands, The Hague, the Netherlands

Balk, Bert, Rotterdam School of Management, Erasmus University, Rotterdam, the Netherlands*

Bashir, Shakila, Forman Christian Collage, Lahore, Punjab, Pakistan

Bates, Nancy, U.S. Census Bureau, Washington, D.C., U.S.A.

Bavdaž, Mojca, University of Ljubljana, Ljubljana, Slovenia

Beaumont, Jean-Francois, Statistics Canada, Ottawa, Canada

Benedetti, Roberto, University of Chieti Pescara, Pescara, Italy*

Beręsewicz, Maciej, Poznań University of Economics and Business, Wielkopolska, Poland*

Beresovsky, Vladislav, National Center for Health Statistics, Hyattsville, Maryland, U.S.A.*

Berglund, Frode, Statistics Norway, Oslo, Norway

Bersimis, Sotiris, University of Piraeus, Piraeus, Greece

Bethlehem, Jelke, Leiden University, Leiden, the Netherlands*

Beyler, Amy, Mathematica Policy Research Health, Arlington, Virginia, U.S.A.

Białek, Jacek, University of Lodz, Lodz, Poland*

Biggeri, Luigi, University of Florence, Florence, Italy*

Bijlsma, Ineke, Maastricht University, Maastricht, the Netherlands* 
Bivand, Roger, NHH Norwegian School of Economics, Bergen, Norway

Bohk-Ewald, Christina, Max Planck Institute for Demographic Research, Rostock, Germany

Bocquier, Philippe, Catholic University of Louvain, Louvain-la-Neuve, Belgium*

Boldsen, Carsten, UNECE, Geneva, Switzerland

Boonstra, Harm Jan, Statistics Netherlands, Heerlen, the Netherlands*

Bottone, Marco, Bank of Italy, Rome, Italy

Braaksma, Barteld, Statistics Netherlands, Utrecht, the Netherlands

Briceno-Rosas, Roberto, GESIS Leibniz Institute for the Social Sciences, Mannheim, Germany*

Brick, Michael, Westat, Rockville, Maryland, U.S.A.*

Breidt, Jay, Colorado State University, Colorado, U.S.A.

Brown, James University of Technology Sydney, Broadway, Australia

Brunåker, Fabian, Valueguard Index Sweden, Uppsala, Sweden*

Brunori, Paulo, University of Bari Aldo Moro, Bari, Italy

Bryant, John, Bayesian Demography Limited, Russley, Christchurch, New Zealand*

Buono, Dario, Eurostat, Mamer, Luxembourg

Burgard, Jan, University of Trier, Trier, Germany*

Cage, Robert, Bureau of Labor Statistics, Washington D.C., U.S.A.

Calviño, Aida, Universitat Rovira i Virgili, Madrid, Spain

Capecchi, Stefania, University of Naples Federico II, Naples, Italy*

Chen, Sixia, Westat, Rockville, Maryland, U.S.A.*

Cheng, Hao, National Academy of Innovation Strategy, Chaoyang, China

Coelho, Edviges, Statistics Portugal, Lisbon, Portugal

Cohen, Robin, National Center for Health Statistics, Hyattsville, Maryland, U.S.A.

Coffey, Stephanie, U.S. Census Bureau, Washington, D.C., U.S.A.*

Conti, Pier Luigi, La Sapienza - University of Rome, Rome, Italy

Coquet, Francois, ENSAI, Bruz, France

Creel, Darryl, RTI International, Rockville, Maryland, U.S.A.*

Cruyff, Maarten, Utrecht University, Utrecht, the Netherlands

Czajka, John, Mathematica Policy Research, Washington. D.C., U.S.A.*

D’Alberto, Riccardo, University of Bologna, Bologna, Italy

Daas, Piet, Statistics Netherlands, Heerlen, the Netherlands

Dalla Chiara, Elena, University of Verona, Verona, Italy

Davidson, Russell, McGill University, Montreal, Quebec, Canada

Davern, Michael, NORC/University of Chicago, Chicago, Illinois, U.S.A.

De Coninck, David, Catholic University of Leuven, Leuven, Belgium*

De Haan, Jan, Statistics Netherlands, The Hague, the Netherlands*

De Leeuw, Edith, University of Utrecht, Utrecht, the Netherlands

Dennis, Michael, National Opinion Research Center, AmeriSpeak, Sunnyvale, California, U.S.A.

Deutsch, Tomi, Zavod Republike Slovenije za šolstvo, Ljubljana, Slovenia

De Waal, Ton, Statistics Netherlands, The Hague, the Netherlands

Di Cecco, Davide, La Sapienza - University of Rome, Rome, Italy*

Di Consiglio, Loredana, Italian National Institute of Statistics, Rome, Italy 
Diewert, Erwin, University of British Colombia, Vancouver, British Columbia, Canada*

Di Fonzo, Tommaso, University of Padova, Padova, Italy*

Di Gennaro, Luca, National Statistics Office, Valletta, Malta*

Di Iorio, Francesca, University of Naples Federico II, Naples, Italy*

Di Zio, Marco, Italian National Institute of Statistics, Rome, Italy

Doidge, James, Intensive Care National Audit and Research Centre, London, UK

Drechsler, Jörg, Institute for Employment Research, Nuremberg, Germany

Dykema, Jennifer, University of Wisconsin, Madison, Wisconsin, U.S.A.*

Eck, Daniel, University of Illinois, Champaign, Illinois, U.S.A.

Eggleston, Jonathan, U.S. Census Bureau, Washington, D.C., U.S.A.

Elliott, Duncan, Office for National Statistics, Newport, UK

Erciulescu, Andreea, National Institute of Statistical Sciences, Washington, D.C., U.S.A.*

Evangelista, Rui, Eurostat, Luxembourg, Luxembourg

Evans, Thomas, U.S. Bureau of Labor Statistics, Washington D.C., U.S.A.

Fabrizi, Enrico, Catholic University, Piacenza, Italy

Falorsi, Piero, Italian National Institute of Statistics, Rome, Italy

Farrugia, Naomi, National Statistics Office Malta, Valletta, Malta

Fischer, Mirjam, German Institute for Economic Research, Berlin, Germany

Fink, Paul, Ludwig Maximilian University of Munich, Munich, Germany

Flower, Tanya, Office for National Statistics, Newport, UK

Fonseca, Thais, University of Warwick, Coventry, UK

Fuller, Wayne, Iowa State University, Ames, Iowa, U.S.A.*

Gemenis, Kostas, Max Planck Institute for the Study of Societies, Cologne, Germany*

Geßendorfer, Jonathan, United Nations Statistics Division, New York, U.S.A.

Giesen, Deirdre, Statistics Netherlands, Heerlen, the Netherlands*

Gile, Krista, University of Massachusetts, Amherst, Massachusetts, U.S.A.

Goicoa, Tomás, Public University of Navarre, Navarre, Spain

Goldhammer, Bernhard, European Central Bank, Frankfurt am Main, Germany

Golinelli, Daniela, RAND Corporation, Santa Monica, California, U.S.A.

Graham, Patrick, Statistics New Zealand, Christchurch, New Zealand*

Grazzini, Jacopo, Eurostat, Luxembourg, Luxembourg

Greselin, Francesca, University of Milan-Bicocca, Milan, Italy

Groenitz, Heiko, Philipps-University Marburg, Marburg, Germany

Gweon, Hyukjun, University of Waterloo, Waterloo, Ontario, Canada

Hanif, Muhammad, National College of Business Administration and Economics, Lahore, Pakistan

Haraldsen, Gustav, Statistics Norway, Kongsvinger, Norway

Haslett, Stephen, Massey University, Palmerston North, Manawatu, New Zealand

He, Yulei, National Center for Health Statistics, CDC, Hyattsville, Maryland, U.S.A.*

Heckathorn, Douglas, Cornell University, Ithaca, New York, U.S.A.

Hedlin, Dan, Stockholm University, Stockholm, Sweden*

Heuchenne, Cedric, University of Liege, Liège, Belgium*

Heumann, Christian, Ludwig Maximilian University of Munich, Munich, Germany

Hill, Robert, University of Graz, Graz, Austria*

Himelein, Kristen, World Bank, Washington, D.C., U.S.A. 
Honchar, Oksana, Australian Bureau of Statistics, Sydney, Australia

Hu, Jingchen, Vassar College, Poughkeepsie, New York, U.S.A.

Humer, Stefan, Vienna University of Economics, Vienna, Austria*

Iseh, Matthew, Akwa Ibom State University,Akwa Ibom, Nigeria*

Jansen, Ronald, UN Statistics Division, New York, U.S.A.*

Jaspers, Eva, Utrecht University, Utrecht, the Netherlands

Johansson, Anton, Statistics Sweden, Örebro, Sweden

Jones, Jacqui, Australian Bureau of Statistics, Belconnen, Australia

Joyce, Patrick, U.S. Census Bureau, Washington, D.C., U.S.A.

Joye, Dominique, University of Lausanne, Lausanne. Switzerland

Junker, Christoph, Federal Statistical Office Health, Neuchâtel, Switzerland

Karlberg, Forough, Luxembourg Statistical Services, Niederanven, Luxembourg*

Karr, Alan, RTI International, Durham, North Carolina, U.S.A.*

Karanka, Joni, Office for National Statistics, Cardiff, UK*

Kavee, Andrew, University of North Carolina at Chapel Hill, Chapel Hill, North Carolina, U.S.A.

Kennedy, Lauren, Monash University, Melbourne, Australia

Kern, Christoph, University of Mannheim, Mannheim, Germany*

Khan, M.G.M, University of the South Pacific, Suva, Fiji*

Kim, Jae-Kwang, Iowa State University, Ames, Iowa, U.S.A.*

Kinyon, David, Energy Information Agency, Washinton, D.C., U.S.A.

Kirby, Graham, University of St. Andrews, St. Andrews, Fife, UK

Klee, Mark, U.S. Census Bureau, Washington, D.C., U.S.A.

Kleven, Øyvin, Statistics Norway, Oslo, Norway

Koerner, Thomas, German Federal Statistical Office, Wiesbaden, Germany

Kolenikov, Stanislav, Abt SRBI, Silver Spring, Maryland, U.S.A.

Konjin, Paul, Eurostat, Luxembourg, Luxembourg

Kott, Phillip, RTI International, Derwood, Maryland, U.S.A.

Kowarik, Alexander, Statistics Austria, Vienna, Austria*

Koyuncu, Nursel, Hacettepe University, Ankara, Turkey

Kristoffersson, Ida, Swedish National Road and Transport Research Institute, Stockholm,

Sweden

Lamboray, Claude, Eurostat, Luxembourg, Luxembourg

Larsen, Michael, George Washington University, Rockville, Maryland, U.S.A.*

Laud, Purushottam, Medical College of Wisconsin, Milwaukee, Wisconsin, U.S.A.*

Lee, Sunghee, University of Michigan, Ann Arbor, Michigan, U.S.A.

Lindholm, Mathias, Stockholm University, Stockholm, Sweden

Lineback, Fane, U.S. Census Bureau, Washington, D.C., U.S.A.

Lipps, Oliver, University of Lausanne, Lausanne, Switzerland*

Little, Roderick, University of Michigan, Ann Arbor, Michigan, U.S.A.*

Liu, Mingnan, SurveyMonkey, Palo Alto, California, U.S.A.*

Loong, Bronwyn, Australian National University Canberra, Australia

Loosveldt, Geert, Catholic University of Leuven, Leuven, Belgium*

Lundquist, Peter, Statistics Sweden, Solna, Sweden

Luiten, Annemieke, Statistics Netherlands, Heerlen, the Netherlands 
MacDonald, Angus, Heriot-Watt University, Edinburgh, UK

MacFeely, Steve, UN Conference on Trade and Development, Geneva, Switzerland* Magnusson, David, Valueguard Index Sweden, Uppsala, Sweden

Maitland, Aaron, National Center for Health Statistics, Hyattsville, Maryland, U.S.A.

Malmros, Jens, Statistics Sweden, Solna, Sweden

Maltagliati, Mauro, University of Florence, Florence, Italy

Maples, Jerry, U.S. Census Bureau, Washington, D.C., U.S.A.*

Maslovskaya, Olga, University of Southampton, Southampton, UK

Massing, Natascha. GESIS Leibniz Institute for the Social Sciences, Mannheim, Germany

Mazzuco, Stefano, Padua University, Padua, Italy

McElroy, Tucker, U.S. Census Bureau, Washington, D.C., U.S.A.*

Mecatti, Fulvia, University of Milan-Bicocca, Milan, Italy

Mercer, Andrew, Pew Research Center, Washington, D.C., U.S.A.

Misson, Sebastian, The Social Research Centre, Melbourne, Australia

Mitra, Robin, University of Southampton, Southampton, UK

Modugno, Lucia, Bank of Italy, Rome, Italy

Moradi, Abbas, Statistical Centre of Iran, Tehran, Iran

Morales Gonzalez, Domingo, Miguel Hernández University of Elche, Elche, Spain

Mothashami, Gholamreza, Ferowsi University of Mashhad, Iran*

Moultrie, Tom, University of Cape Town, Rondebosch, South Africa

Mukherjee, Diganta, Indian Statistical Institute, Kolkata, India*

Mukhopadhyay, Pushpal, SAS Institute Inc., Cary, North Carolina, U.S.A.

Mule, Vincent, U.S. Census Bureau, Suitland, Maryland, U.S.A.

Neri, Laura, University of Siena, Siena, Italy*

Neumann, Robert, Dresden University of Technology, Dresden, Germany

Nicholson, James, Durham University, Durham, UK

Nishimura, Raphael, Abt SRBI, Los Angeles, California, U.S.A.*

Norberg, Anders, Statistics Sweden, Solna, Sweden

Ograjensek, Irena, University of Ljubljana, Ljubljana, Slovenia*

Olteanu-Raimond, Ana-Maria, IGN, Saint-Mandé, France

Oncel Cekim, Hatice, Hacettepe University, Ankara, Turkey

Ogwang, Tomson, Brock University, St. Catharines, Ontario, Canada

Opsomer, Jean, Westat, Rockville, Maryland, U.S.A.

Oral, Evrim, Louisiana State University, New Orleans, Louisiana, U.S.A.

Orusild, Tiina, Statistics Sweden, Solna, Sweden

Osier, Guillaume, National Institute of Statistics and Economic Studies, Luxembourg, Luxembourg

Palm, Viveka, Statistics Sweden, Solna, Sweden

Pang, Osbert, U.S. Census Bureau, Washington, D.C., U.S.A.

Park, Mingue, Korea University, Seoul, Republic of Korea

Park, Minjeong, Statistical Research Institute, Seo-gu, Daejeon, Republic of Korea

Pedlow, Steven, NORC/University of Chicago, Chicago, Illinois, U.S.A.

Persson, Andreas, Statistics Sweden, Örebro, Sweden*

Pinheiro Jacob, Guilherme, Nossa Sra. das Graças, Manaus, Amazonas, Brazil

Planas, Christophe, Joint Research Centre of EC, Ispra, Varese, Italy* 
Pascal, Joanne, U.S. Census Bureau, Washington, D.C., U.S.A.

Pratesi, Monica, University of Pisa, Pisa, Italy.

Presser, Stanley, University of Maryland, College Park, Maryland, U.S.A.

Proietti, Tommaso, University of Rome, Rome, Italy*

Psarakis, Stelios, University of Athens, Athens, Greece*

Quick, Harrison, Drexel University, Philadelphia, Pennsylvania, U.S.A.

Rambaldi, Alicia, University of Queensland, Brisbane, Australia

Rau, Roland, Max Planck Institute for Demographic Research, Rostock, Germany

Raymer, James, Australian National University, Canberra, Australia*

Righi, Paolo, Italian National Institute of Statistics, Rome, Italy*

Robbins, Michael, University of Missouri, Columbia, Missouri, U.S.A.

Roberson, Andrea, U.S. Census Bureau, Washington, D.C., U.S.A.*

Robison, Edwin, U.S. Bureau of Labor Statistics, Washington D.C., U.S.A.

Rocco, Emilia, University of Florence, Florence, Italy

Rothe, Patrick, Bavarian State Office for Statistics, Fuerth, Germany*

Rothschild, David, Microsoft Research, New York, New York, U.S.A.

Russ, Daniel, National Institutes of Health, Bethesda, Maryland, U.S.A.

Salvati, Nicola, University of Pisa, Pisa, Italy

Scannapieco, Monica, Italian National Institute of Statistics, Rome, Italy

Scheffer, Fredrik, Statistics Sweden, Solna, Sweden*

Scherpenzeel, Annette, Technical University of Munich, Munich, Germany

Schliep, Erin, University of Missouri, Columbia, Missouri, U.S.A.

Schmid, Timo, Free University of Berlin, Berlin, Germany*

Schmidt, Tobias, Deutsche Bundesbank, Frankfurt am Main, Germany

Scholtus, Sander, Statistics Netherlands, The Hague, the Netherlands

Schonlau, Matthias, University of Waterloo, Waterloo, Ontario, Canada*

Schoumaker, Bruno, Catholic University of Louvain, Louvain-la-Neuve, Belgium

Schouten, Berry, Statistics Netherlands, The Hague, the Netherlands

Sebastiani, Fabrizio. Qatar Computing Research Institute, Doha, Qatar

Sengupta, Manisha, National Center for Health Statistics, CDC, Hyattsville, Maryland, U.S.A.*

Serfioti, Maria. European Commission (DG ESTAT), Luxembourg, Luxembourg

Seyb, Allyson, Statistics New Zealand, Christchurch, New Zealand

Shabbir, Javid, Quaid-i-Azam University, Islamabad, Pakistan*

Shin, Hee-Choon, National Center for Health Statistics, Hyattsville, Maryland, U.S.A.*

Si, Yajuan, University of Michigan, Ann Arbor, Michigan, U.S.A.

Sief, Asghar, Bu-Ali Sina University, Hamedan, Iran

Silver, Mick, International Monetary Fund, Washington, D.C., U.S.A.

Singh, G.N., Indian Institute of Technology, Dhanbad, India

Singh, Sarjinder, Texas AM University-Kingsville, Kingsville, Texas, U.S.A.

Sixta, Jaroslav, University of Economics, Prague, Czech Republic

Smith, Duncan, University of Manchester, Manchester, UK*

Smith, Peter, University of Southampton, Southampton, UK

Sparapani, Rodney, Medical College of Wisconsin, Milwaukee, Wisconsin, U.S.A.*

Spoorenberg, Thomas, United Nations Population Division, New York, U.S.A. 
Steinmetz, Stephanie, University of Lausanne, Lausanne, Switzerland

Steurer, Miriam, University of Graz, Graz, Austria

Ståhl, Olivia, Statistics Sweden, Solna, Sweden

Stinson, Martha, U.S. Census Bureau, Washington, D.C., U.S.A.

Stockhammar, Pär, Stockholm University, Stockholm, Sweden*

Stoop, Ineke, SCP Sociaal en Cultureel Planbureau, The Hague, the Netherlands

Struminskaya, Bella, Utrecht University, Utrecht, the Netherlands

Szymkowiak, Marcin, Poznan University of Economics and Business, Wielkopolska, Poland

Tanguy, Antoine, National Statistics Office, Valletta, Malta

Templ, Matthias, Statistics Austria, Vienna, Austria

Ten Bosch, Olav, Statistics Netherlands, The Hague, the Netherlands

Tent, Reinhard, Federal Statistical Office, Wiesbaden, Germany

Thalji, Lisa, RTI International, Research Triangle Park, North Carolina, U.S.A.

Thompson, Mary, University of Waterloo, Waterloo, Ontario, Canada

Tijdens, Kea, University of Amsterdam, Amsterdam, the Netherlands

Todorov, Valentin, UNIDO, Research and Statistics Vienna International Centre, Vienna, Austria

Tomadini, Andrew, Australian Bureau of Statistics, Belconnen, Australia

Tuoto, Tiziana, Italian National Institute of Statistics, Rome, Italy*

Umbach, Gaby, European University Institute, Florence, Italy*

Valliant, Richard, University of Michigan, Chevy Chase, Maryland, U.S.A.

Van den Brakel, Jan, Maastricht University School of Business and Economics,

Maastricht, the Netherlands

Van der Laan, Jan, Statistics Netherlands, The Hague, the Netherlands

Van Loon, Ken, Statistics Belgium, Brussels, Belgium

Verma, Med Ram, Indian Veterinary Research Institute, Izatnagar, Bareilly, Uttar

Pradesh, India*

Verzosa, Nina, University of Sydney, Sydney, Australia

Von Auer, Ludwig, University of Trier, Trier, Germany

Vozar, Ondrej, Czech Statistical Office, Prague, Czech Republic

Waldmann, Patrik, Swedish University of Agricultural Sciences, Uppsala, Sweden*

Walter, Paul, Free University of Berlin, Berlin, Germany

Wang, Yan, RMIT University, Melbourne, Australia*

Warren, John, University of Minnesota, Minneapolis, Minnesota, U.S.A.

Watson, Nichole, University of Melbourne, Melbourne, Australia

Wesolowski, Jacek, Central Statistical Office, Warszawa, Poland

Willenborg, Leon, Statistics Netherlands, The Hague, the Netherlands

Wiśniowski, Arkadiusz, University of Southampton, Southampton, UK

Wright, Tommy, U.S. Census Bureau, Washington, D.C., U.S.A.

Wu, Changbao, University of Waterloo, Waterloo, Ontario, Canada

Yildiz, Dilek, JOS-2019-0124, University of Southampton, Southampton, UK

Zabala, Felipa, Statistics New Zealand, Wellington, New Zealand

Zammit, Silvan, National Statistics Office, Valletta, Malta

Zenga, Michele, University of Milan-Bicocca, Milan, Italy 
Zhang, Junni, Peking University, Beijing, China

Zhang Mark (Xichuan), Australian Bureau of Statistics, Belconnen, Australia

Zhang, Yunxi, University of Mississippi; Jackson, Mississippi, U.S.A.*

Zimmermann, Thomas, Federal Statistical Office, Wiesbaden, Germany 